\title{
RECUPERAÇÃO DAS VÍTIMAS DE TRAUMATISMO CRÂNIO-ENCEFÁLICO NO PERÍODO DE 1 ANO APÓS O TRAUMA
}

\author{
Regina Marcia Cardoso de Sousa* \\ Maria Sumie Koizumi**
}

SOUSA,R.M.C.de:KOIZUMI,M.S., Recuperação das vítimas de traumatismo crânio-encefálico no período de 1 ano após o trauma. Rev.Esc.Enf.USP,v.30,n.3,p.484-500,dez. 1996.

Estudo prospectivo longitudinal sobre a recuperação aos 12 meses, de vitimas de traumatismo crânio-encefálico (TCE) de diferentes gravidades, com idade entre 12 e 60 anos. As vítimas foram avaliadas 1 ano após o trauma considerando-se tanto suas limitações funcionais mensuradas pela Escala de Resultados de Glasgow (ERG) em sua versão de oito categorias, como também, o seu retorno à produtividade. Aos 12 meses, $77,2 \%$ das vitimas alcançaram a pontuação 0 e 1 na ERG ampliada. Destas, 38,6\% obtiveram a pontuação 0 , ou seja, recuperação total. Indivíduos incapazes (pontuação $>1$ ) foram $22,8 \%$ sendo aqueles com incapacidade moderada (+), ERG2, e grave (+), ERG4, os mais freqüentes. Retorno à produtividade ocorreu em $83,3 \%$ das vitimas e destas, $19,4 \%$ tinham alterações na ocupação principal.

UNITERMOS: Trauma crânio-encefálico. Recuperação p6s-traumática. Escala de Resultados de Glasgow.

No Brasil, as mortes por acidente e violência tomaram um grande vulto nos últimos anos e o trauma é atualmente uma das mais freqüentes e importantes causas de mortalidade geral e morbidade ${ }^{30}$. Embora as estatísticas não apresentem dados especificos de mortalidade e morbidade por trauma ou trauma crânio-encefálico (TCE) ${ }^{31,39}$, sabe-se que as causas externas eram em 1989 a terceira causa de mortalidade geral no país, e segunda causa de mortalidade no estado e município de São Paulo

A morbidez por TCE é um dos itens que, na revisão da literatura nacional sobre trauma, apresenta poucas informações e que nos estudos internacionais deixa questionamentos, principalmente no que se refere as características e duração das incapacidades resultantes do TCE de diferentes gravidades, assim como de fatores que influenciam quantitativamente e qualitativamente a recuperação.

\footnotetext{
* Professor Doutor do Departamento de Enfermagem Médico-Cirúrgica da Escola de Enfermagem da USP.

* Professor Titular do Departamento de Enfermagem Médico-Cirúrgica da Escola de Enfermagem da USP.
} 
$O$ interesse para o desenvolvimento de trabalhos, especificamente nesta área, é acrescido pela freqüência e repercussão das alterações presentes nos pacientes após TCE e pela expectativa de um aumento do número de sobreviventes de trauma cerebral. Ainda que a introdução de novas técnicas e tratamentos tenha diminuído a intensidade das incapacidades, estes avanços aumentaram também de forma importante a sobrevida após acidente ou violência ${ }^{2}$. Este fato somado à grande incidência do trauma, traz a expectativa de um aumento do número de sobreviventes com deficiências residuais, que afetam o retorno para o estado funcional anterior ao trauma"l.

Com a finalidade de melhor avaliar o ônus social, familiar e individual de uma vítima de TCE, parâmetros têm sido utilizados com os quais se procura traduzir as conseqüências do trauma na vida quotidiana do traumatizado.

Desta forma, a habilidade do traumatizado em realizar atividades da vida diária, viver independentemente, reassumir responsabilidades anteriores e retornar ao trabalho ou a ocupação principal têm sido informações usadas para avaliar o padrão de recuperação pós-trauma ${ }^{42}$.

Para nomear a situação, em que se procura considerar a capacidade do indivíduo como um todo funcional e integrado na avaliação de indivíduos sobreviventes do TCE, diferentes terminologias são utilizadas, sendo as mais freqüentemente encontradas: medir os resultados do TCE; avaliar a saída funcional ou a recuperação funcional pós-traumática. Tais denominações são utilizadas para designar situações onde se visa verificar ou medir quanto o evento do TCE alterou a capacidade do indivíduo desempenhar suas funções e desempenhar papeis sociais que dele são esperados. No transcorrer deste estudo, a terminologia avaliar a saída funcional será utilizada para se referir a essa situação.

No intuito de quantificar a capacidade do indivíduo, como um todo funcional e integrado, algumas escalas têm sido propostas ${ }^{13,21}$, sendo a mais usada a Escala de Resultados de Glasgow (ERG) ${ }^{13.15 .43}$. Esta escala, foi elaborada por JENNETT e BOND, para avaliação da saída funcional após dano encefálico, inclusive os decorrentes de lesões traumáticas, e como tal, considera a heterogeneidade das incapacidades físicas e psicológicas presentes nas vítimas de TCE, assim como o efeito de tais incapacidades sobre a função social ${ }^{21,43}$.

$\mathrm{Na}$ descrição do uso da $\mathrm{ER}\left(\mathrm{x}^{21}\right.$, retorno à produtividade é uma circunstância que não deve ser considerada para a classificação do indivíduo. Segundo seus autores, JENNETT e BOND, essa ocorrência não é um índice realista de saída funcional, à medida que pode ocasionar falsas impressões. Situações socioeconômicas da comunidade podem dificultar o retorno do enfermo para a produtividade, mesmo apresentando recuperação total. Por outro lado, alguns pacientes, com considerável incapacidade, podem retornar a sua ocupação principal, mesmo porque tal ocupação é compatível com aqu ela incapacidade ${ }^{21,23}$.

Entretan to é inegável que o primeiro interesse em alcançar a recuperação conseqüente a uma doença ou trauma é retornar o indivíduo a sua maior atividade social esperada, isto é retornar ao emprego, à escola ou manter atividades domésticas ou outra relevante ${ }^{28}$. 
Nesse sentido, outro aspecto a ser considerado é que o trabalho remunerado é tradicionalmente vis to na sociedade moderna como proposta central de vida, tomando para si, papel principal para suprir tanto necessidades de auto-estima como econômicas ${ }^{11}$.

Frente a essa dualidade, em que se aceita, por um lado, os motivos alegados por JENNETT e BOND para não considerar retorno à produtividade como indicador de saída funcional após TCE, e por outro, reconhece-se os padrões sociais que impõem o trabalho como componente essencial para medida dos resultados do trauma, vários autores têm optado por uma alternativa conciliatória em que a avaliação da saída funcional do trauma é acrescida de dados sobre retorno à produtividade ${ }^{11,14,18,24,27,28,32,35,36,37}$.

Acredita-se, portanto, que para uma análise mais abrangente dos resultados sociais e pessoais do TCE, em pacientes sobreviventes, cabe, além do uso da ERG, dados complementares sobre o retorno à produtividade no período que se segue ao evento trauma.

Propõe-se como objetivo para o presente estudo, caracterizar o padrão de recuperação da vítima de TCE no período de 1 ano pós-trauma, avaliando-se saída funcional e retorno a produtividade.

\section{Metodologia}

A população de estudo foi constituída por vítimas de TCE admitidas para tratamento no Instituto Central do Hospital das Clínicas da Faculdade de Medicina da Universidade de São Paulo (HCFMUSP) no período de março a junho de 1993, em decorrência de acidente ou violência ocorridos nesse mesmo período.

Os critérios de inclusão das vítimas foram: ter entre 12 e 60 anos de idade; apresentar dados registrados no seu prontuário ou cadastro geral do hospital para sua localização; residir na Grande São Paulo; comparecer ao ambulatório por convocação e consentir em participação da pesquisa.

Pacientes entre 12 e 60 anos foram incluídos, considerando-se esta uma faixa etária em que os indivíduos normalmente têm independência para as atividades básicas de sua manutenção.

O critério de inclusão de vítimas residentes na Grande São Paulo foi fixado para evitar deslocamento de vítimas de distâncias maiores que a de tal perímetro e para otimizar a probabilidade de comparecimento por convocação.

Excluíram-se aqueles que solicitaram alta a pedido ou evadiram-se do hospital devido à baixa possibilidade de sua aderência a um estudo de seguimento e a possibilidade de diferentes conseqüências do trauma em vítimas que não completaram tratamento na fase aguda pós -traumática. Foram também excluídos vítimas que apresentaram reincidencia de trauma durante o periodo de seguimento deste estudo, uma vez que, esse fato dificultaria delimitar conseqüências específicas de cada um dos eventos traumáticos. 
Os dados das vítimas foram obtidos, inicialmente, na lista fornecida pela Divisão de Arquivo Médico, confirmados pelos dados do prontuário de cada paciente e pela avaliação individual da vítima feita por uma das pesquisadoras, num dos consultórios do ambulatório de Trauma de Crânio, durante o período de setembro de 1993 a agosto de 1994 .

Da lista da Divisão de Arquivo Médico constaram, além da informação referente ao tipo de TCE, penetrante ou fechado, os seguintes dados: número de registro do paciente no hospital; idade; sexo; óbito ocorrido na internação; tempo de permanência hospitalar e data de internação e alta.

Nesta lista foram selecionados os pacientes sobreviventes, internados nos meses de março, abril, maio e junho de 1993, das faixas etárias de 12 a 60 anos.

Feita esta primeira seleção, iniciou-se o processo de busca de prontuários através do número do registro geral dos pacientes no hospital. Localizado o prontuário e confirmados os demais dados de seleção do indivíduo, foi enviado carta para cada um destes pacientes, solicitando comparecimento ambulatorial, em data marcada entre 6 e 8 meses apcs a ocorrência do trauma. Neste período era realizada uma la avaliação desde que o paciente concordasse em participar do estudo e era verificada a disponibilidade das vítimas para participar de uma $2 a$ avaliação entre 12 e 14 meses apos o trauma, período em que os pacientes também foram notificados através de carta para comparecimento ambulatorial. O presente relatório de pesquisa refere-se basicamente as avaliações realizadas nesse $2^{\circ}$ período.

Quanto à convocação de pacientes, determinou-se ainda que quando ocorria a falta em primeira convocação uma segunda carta para comparecimento ambulatorial era enviada. Nos casos em que não havia comparecimento, mesmo perante as duas correspondências, o paciente era automaticamente eliminado do seguimento pós-traumático. Quando havia menção de número telefônico em alguma das fontes de dados do paciente, este também era um dos meios utilizados para localização das vítimas. Somente os pacientes internados na época da avaliação eram examinados nas instituições e os impossibilitados de comparecer ao ambulatório, no próprio domicílio.

Como instrumento de medida de saída funcional, utilizou-se a ERG na sua versão ampliada ${ }^{2}$. A opção pelo uso desse instrumento foi conseqüente a sua maior sensibilidade para detectar alterações apresentadas na condição das vítimas, quando comparado com a ERG original. Outro fator que contribuiu para essa opção foi a possibilidade de com o uso da ERG ampliada, realizar-se comparações com resultados de estudos similares que vêm usando freqüentemente a $E R G$ original como parâmetro de avaliação da saída funcional das vítimas de TCE. Como pode ser observado, na descrição dessa escala, a transposição de categorias da ERG ampliada para a classificação da ERG original é bastante simplificada à medida que a primeira apresenta categorias adicionais que são subcategorias de sua versão original.

A escala original, publicada em $1975^{21}$, contém 5 categorias para classificação dos pacientes: morte; estado vegetativo persistente; incapacidade grave; incapacidade moderada e boa recuperação. 
Mesmo tendo sido largamente utilizada nestes últimos 20 anos na sua forma original, tem se reconhecido na ERG insuficiência em refletir quantitativamente mudanças sutis dos pacientes. Esta limitação foi reconhecida pelos próprios autores que sugeriram uma subdivisão da escala original para permitir uma maior sensibilidade na avaliação de pacientes que retomam a consciência ${ }^{23}$.

Desta forma, dentro das categorias originais dos indivíduos conscientes (incapacidade grave, incapacidade moderada e boa recuperação), foi proposta uma subdivisão dos indivíduos com melhor ou pior nivel de recuperação. A escala passou então a apresentar 8 categorias, sendo que 6 graduações da escala passaram a ser utilizadas para designar a condição do indivíduo que retornou à consciência após trauma ${ }^{5,23}$.

Apresenta-se a seguir a ERG ampliada juntamente com os critérios de pontuação. Como o objetivo do estudo foi a classificação apenas de pacientes sobreviventes, a pontuação 7 (sete) que é utilizada em situações de morte foi excluida.

Boa recuperação (+): nesta categoria foram incluídos indivíduos que reassumiram vida normal sem nenhuma alteração ou queixa conseqüente ao trauma. Retorno à produtividade não foi um parâmetro básico para inclusão nesta categoria.

( 1 ) Boa recuperação (-): refere-se a indivíduos que reassumiram vida normal com presença de leves deficiências físicas ou mentais. Da mesma forma que na categoria anterior, o retorno à produtividade não foi parâmetro básico para inclusão na categoria.

( 2 ) Incapacidade moderada (+): "independente mas incapaz". Podem realizar as atividades básicas do autocuidado* $\mathrm{e}$ as atividades básicas para manutenção da independência** sem ajuda, por si próprio, com ou sem dificuldades na execução. () ponto básico de diferenciação para boa recuperação é que os indivíduos que são incluídos nessas categorias não conseguem retomar todas as atividades realizadas anteriormente ao trauma.

* Atividades básicas da vida diária ou do autocuidado, tem-se apresentado. em escalas de avaliação de saída funcional, aquelas atividades que estão limitadas ao cuidado pessoal e referem-se à: alimentar-se, banhar-se, manter os cuidados referentes à função excretora, vestir-se e deslocar-se dentro do cômodo o qual se encontra $7,16,23.34$.

Atividades básicas para manutençáo da independéncia, englobam as tar efas de autocuidado. porém, elas envolvem também outras atividades e comportamentos que são considerados como componentes essenciais da vida quotidiana, os quais estáo relacionados a seguir: ouvir; ver; expressar-se verbalmente; entender mensagens; deslocar-se dentro de casa; deslocar-se pela vizinhança - sem uso de transportes; deslocar-se - fazendo uso de transportes públicos: transpor desníveis - subir ou descer degraus; preparar refeiçóes: cuidar de roupas - passar, lavar, guardar; manter a limpeza doméstica, carregar objetos e produtos necessários àl subsistência; fazer compras - manejar dinheiro; evitar acontecimentos que lesem a integridade física; registrar e compreender as relações entre os objetos e as pessoas em situaçóes cotidiana; adquirir novos conhecimentos; planejar suas atividades na vida cotidiana; manter comportamentos sociais aceitos. Os itens que constam nessa listagem de atividades foram determinados a partir de análise da listagem de capacidades apresentada no Manual para classificação das consequéncias das doenças apresentado pela Organizaçăo Mundial de Saúde $e^{33}$. 
( 3 ) Incapacidade moderada (-): "independente mas incapaz". Pode realizar as atividades básicas do autocuidado e as atividades básicas para manutenção da independência, com ajuda de dispositivos ou em meio ambiente em que há modificações para possibilitar sua realização.

(4) Incapacidade grave (+): "consciente mas dependente". É capaz de manter as atividades básicas do autocuidado, sem ajuda de outra pessoa. Porém, nas atividades básicas para manutenção da independência deve apresentar pelo menos uma atividade que necessita de assistência de outrem.

( 5 ) Incapacidade grave (-): "consciente mas dependente". Não consegue manter as atividades básicas do autocuidado sem auxílio de outra pessoa.

(6) Estado vegetativo persistente: não demonstra evidência de capacidade de elaboração de resposta significativa. Não obedece aos comandos simples e nem pronuncia qualquer palavra. Diferenciar de outras condições em que se tem extrema redução da capacidade de resposta.

Outro critério para avaliar as conseqüências do trauma foi a analise da produtividade. Produtividade pós-trauma foi definida como retorno ao emprego, escola ou afazeres do lar, considerando-se assim como MORRIS et al ${ }^{32}$ essas três atividades de essencial importância para produção nacional à medida que contribuem ou dão estrutura para manutenção da produção de bens em uma sociedade.

Fizeram-se necessárias para possibilitar a avaliação do retorno à produtividade pós-trauma, a categorização das vítimas quanto à ocupação principal e à análise da condição ocupacional pós-trauma.

Através de dados da avaliação, cada paciente era classificado de acordo com sua ocupação principal. Ocupação principal foi então definida como a mais importante atividade exercida antes do TCE, referida pelo paciente. Devido à dificuldade em estabelecer prioridades entre atividades das vitimas estudantes e com ocupação remunerada, ambas as atividades foram consideradas conjuntamente como ocupação principal quando assim presentes. Dentro dessa classificação estabeleceu-se então as seguintes categorias: ocupação remunerada; estudantes; estudantes que também mantinham ocupação remunerada; aposentados e desempregados.

A condição p6s-trauma, em relação à ocupação principal, foi classificada, tendo como base o retorno à produtividade, ou seja, atividade interrompida, alterada devido ao trauma e retorno com o mesmo desempenho.

Quanto à gravidade da lesão ou do trauma e para avaliação do TCE, foram utilizados os seguintes índices, de acordo com a classificação e codificação preconizada pelos seus autores: Escala de Coma de Glasgow (ECGl) ${ }^{44}$; Abbreviated Injury Scale (AIS) ${ }^{1 /}$ Injury Severity Score (ISS) ${ }^{1}$. 
A AIS é um sistema avaliativo, de base anatômica que classifica cada lesão decorrente de um trauma, por região corporea e estabelece um escore que indica gravidade da lesão específica. Apresenta uma escala ordinal de 6 pontos, oscilando de AIS 1 (lesão leve) a AIS 6 (lesão quase sempre fatal). Pela AIS obtém-se o escore de gravidade de uma lesão isoladamente.

A gravidade global do trauma é obtida pelo ISS, escore matematicamente calculado a partir da AIS. Para cálculo do ISS, é estabelecido o escore mais alto para cada uma das regiões corporais. Calcula-se então o ISS, que é a soma dos quadrados dos escores AIS mais altos das três regiões diferentes do corpo mais gravemente traumatizadas.

Com relação ao ISS, os traumatizados têm sido subdivididos em 3 grupos: aqueles com valor de 1 a 15 ; de 16 a 24 e $\geq 25$. Nessa subdivisão, pacientes com intervalos de ISS entre 1 a 15 têm sido categorizados como vítimas de trauma leve, de 16 a 24 como trauma moderado e $\geq 25$ como trauma grave $e^{0,49}$.

Para avaliar especificamente a gravidade do TCE, utilizou-se a ECGl Embora a ECGl fosse elaborada para prover estruturada avaliação clínica do nível de consciência dos pacientes com dano cerebral, ela tornou-se importante parâmetro indicador da gravidade do trau ma cerebral, permitindo comparações entre diferentes pesquisas realizadas ${ }^{20.2225,41.46}$. Escores da ECGl entre 13 e 15 indicam função cerebral próxima a normal, e estes valores têm sido usados para categorizar indivíduos com TCE leve. Observações na escala que somam 8 pontos ou menos geralmente definem coma ${ }^{19}$, o que caracteriza um grupo de vítimas gravemente traumatizado. Pontuação da ECGl entre 9 e 12 representam aquelas vítimas de TCE de gravidade intermediária ${ }^{2037.38}$.

Os dados deste estudo estão apresentados em freqüência absoluta e relativa sendo mostrados em forma de tabelas e quadro.

\section{Resultados, Discussão e Conclusões}

\section{Populacão estudada}

Entre março e junho de 1993, 385 vítimas de trauma com diagnóstico de TCE foram admitidas no Instituto Central do HCFMUSP. Destas, 73 (19,0\%) morreram antes da alta hospitalar; $65(16,9 \%)$ eram sobreviventes menores de 12 anos e $34(8,8 \%)$ sobreviventes maiores de 60 anos. Vítimas sobreviventes com idade entre 12 e 60 anos eram 213. Dessas 213 vítimas, 108 participaram do estudo e 105 não,

Das 108 vítimas de TCE que participaram da primeira fase do estudo, 23 $(21,3 \%)$ tiveram pontuação 0 na $E R G$, ou seja, aos 6 meses apresentaram recuperação total do trauma. Os demais 85 participantes dessa $1^{0}$ fase do estudo foram convocados para $2^{0,}$, realizada entre 12 e 14 meses pós-trauma. Desses 85 
individuos, $60(70,6 \%)$ atenderam a solicitação de comparecimento para $2^{0}$ avaliação, uma vítima foi assassinada nesse período, e 24 indivíduos não compareceram.

Desta forma, conheceu-se a recuperação pós-traumática no período de 1 ano de 83 indivíduos (39,0\% dos 213 sobreviventes entre 12 e 60 anos de idade), sendo 60 , os avaliados 1 ano após o trauma e 23 os que atingiram recuperação total aos 6 meses.

Os 83 participantes que se conheceu a recuperação após 1 ano do trauma tiveram como principais caracteristicas as descritas a seguir.

- Apresentar diferentes níveis de gravidade do trauma, estabelecido pelo sistema AIS/ISS: $44,6 \%$ trauma leve; $43,4 \%$ moderado e $12,0 \%$ grave.

- Ter diferentes níveis de alteração de consciência na primeira avaliação feita pela equipe de neurocirurgia: 8,4\% com ECGl $\leq 8 ; 15,6 \%$ com ECGl entre 9 e $12 ; 72,3 \%$ com $\mathrm{ECGl} \geq 13$.

- As vítimas tinham idade entre 12 e 60 anos sendo que a maioria, $69,9 \%$, tinha até 35 anos.

Saída funcional após 1 ano

Resultados obtidos na ERG ampliada por essas vítimas, são apresentados na Tabela 1 .

Tabela 1 - Distribuição das vítimas com evolução de 1 ano segundo classificação na ERG ampliada. São Paulo, 1993/94.

\begin{tabular}{lc|cc}
\hline ERG ampliada & & $\mathbf{n}^{\circ}$ & $\%$ \\
\hline Categoria & Escore & & \\
. boa recuperação (+) & 0 & 32 & 38,6 \\
boa recuperação (-) & 1 & 32 & 38,6 \\
. incapacidade moderada (+) & 2 & 08 & 9,6 \\
. incapacidade grave (+) & 4 & 08 & 9,6 \\
.incapacidade grave (-) & 5 & 01 & 1,2 \\
.incapacidade grave (+) & $*^{4}$ & 02 & 2,4 \\
\hline TOTAL & & 83 & 100,0 \\
\hline
\end{tabular}

* Vítimas com incapacidade anterior ao trauma.

Como mostra a Tabela 1, no período de 1 ano após trauma, o número de vítimas com recuperação total (escore 0) era igual ao daquelas que embora alcançassem boa recuperação, mantinham queixas ou défices leves que as classificavam em escore 1 . O total de vítimas com boa recuperação foi de $77,2 \%$.

Indivíduos independentes, que necessitavam de adaptações ambientais e artefatos para manutenção da sua independência (escore 3), não foram 
encontrados no periodo de 1 ano apos trauma, assim como indivíduos em estado vegetativo persistente (escore 6).

Dos individuos com incapacidade grave, somente 1 apresentava ainda 1 ano pós-trauma, dependência para atividades básicas de autocuidado. Este, embora obedecesse a ordens simples, apresentava afasia da expressão, déficit motor com predomínio à direita e era um paciente acamado, completamente dependente para atividades básicas do autocuidado, sendo mantido traqueostomizado, com sonda vesical de demora e sonda nasoenteral para alimentação. Diferenças significativas não foram observadas em suas condições 6 meses e 1 ano pos-trauma, sendo esse indivíduo mantido internado desde o trauma até o período da última avaliação desta pesquisa.

Indivíduos dependentes com pontuação 4 na escala eram ainda $12,0 \%(9,6 \%$ $+2,4 \%$ ) no período de 1 ano após trauma, sendo que o percentual total dos dependentes foi de $13,2 \%$ (pontuação $\geq 4$ ).

Vítimas com incapacidades anterior ao trauma eram duas e mantinham no período de avaliação do estudo o mesmo nível de capacidade funcional apresentado anteriormente ao trauma.

No geral, no período de 1 ano, indivíduos com incapacidade moderada (+), ERG igual a 2, e grave (+), ERG igual a 4, foram os mais freqüentes entre os incapazes.

Os resultados da ERG original referentes às publicações que avaliam saída funcional, fixando um período de 1 ano para essa avaliação são apresentados no Quadro 1 e comparados com o presente estudo. Para tal comparação, resultados da ERG ampliada deste estudo foram transformados em categorias da ERG original. Os indivíduos com incapacidade grave $(+)$, anterior ao trauma, foram computados juntamente com as outras vítimas com a mesma categorização, visto que não há menção de exclusão desses indivíduos nos outros estudos apresentados. 


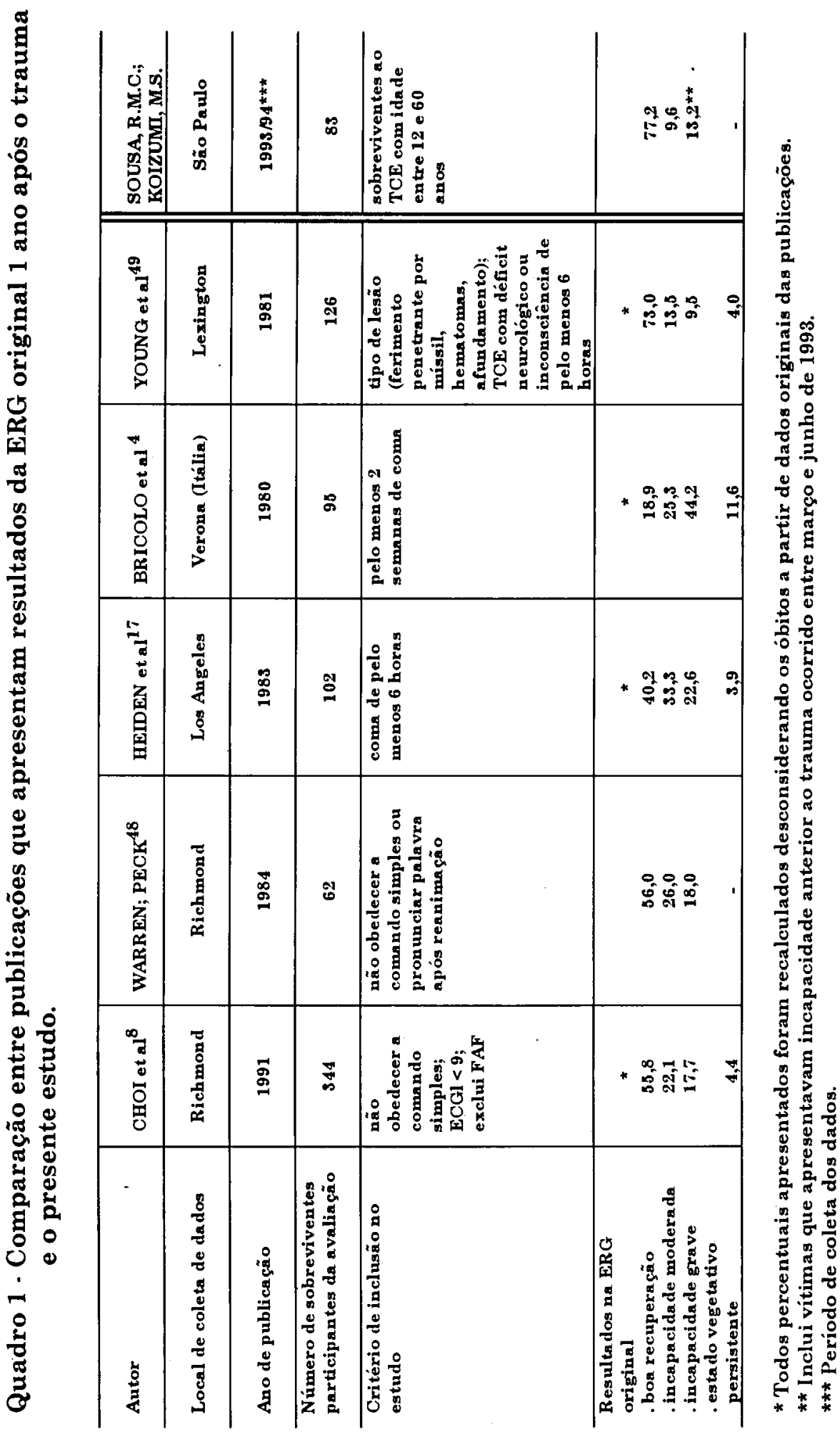


No Quadro 1, observa-se nos critérios de inclusão das publicações analisadas, que quanto à gravidade do TCE, embora todos as publicações sejam de vítimas de TCE grave, condições diferentes foram impostas em dois grupos. $\mathrm{O}$ de $\mathrm{BRICOLO}$ et $\mathrm{al}^{4}$ incluíram vítimas "especialmente graves" à medida que se restringiu a indivíduos que permaneceram em estado de coma por pelo menos 2 semanas e o de YOUNG et al $^{49}$ que, embora tenham avaliado vítimas graves, acrescenta em sua população elementos com lesões anatômicas específicas as quais recebem diferentes categorizações de gravidade no Manual da AIS-90: ferimento penetrante por míssil - AIS $=5$, hematoma intracraniano sem outras especificações - AIS $=4$ e afundamento de crânio - AIS = 3. Embora, conforme referência dos próprios autores, a gravidade do dano cerebral fosse variada, tinham sido operados ou tratados em unidade de terapia intensiva.

No presente estudo, que foram incluídos indivíduos hospitalizados que apresentavam diferente gravidade de trauma craniano e que somente $8,4 \%$ das vítimas apresentaram TCE grave ou $E C G I \leq 8$, o padrão de recuperação das vítimas foi muito semelhante a de YOUNG et al ${ }^{49}$, conforme pode-se observar nos resultados da ERG (vide Quadro 1). A similaridade de resultados é bastante grande principalmente quando comparado com os demais. A associação entre a gravidade inicial do trauma nas suas conseqüências é fato irrefutável, sendo motivo de análises mais detalhadas em outro estudo ${ }^{40}$.

Observa-se ainda no Quadro 1, que excluindo-se o grupo de "especial gravidade" de BRICOLO et $\mathrm{al}^{4}$, o grupo do Centro Médico de Los Angeles, avaliado por HEIDEN et $\mathrm{al}^{17}$, 6 o que apresenta piores conseqüências do trauma. Ainda que os critérios de inclusão desse estudo de Los Angeles assemelhe-se aos de Richmond, no geral foram melhores os resultados apresentados pelas publicações dessa última localidade (CHOI et $\mathrm{al}^{8}$ e WARREN; PECK ${ }^{48}$. Uma diferença na gravidade das vítimas de TCE grave entre a população atendida nesses centros pode estar envolvida nessa diferença de resultados. Estudos têm indicado que indivíduos com TCE grave com valores na ECGl de 3 a 5 têm piores resultados do que aqueles com escores entre 6 e $8^{18,26}$.

No presente estudo, aos 12 meses, $38,6 \%$ de vítimas encontravam-se completamente recuperadas. Entretanto, os incapazes eram ainda $22,8 \%$ e os que mantinham dependência de outrem $13,2 \%$. O ônus social e individual dessas vítimas são ainda bastante acentuados no período de 1 ano, principalmente quando considera-se a gravidade do TCE das vítimas avaliadas.

\section{Retorno à produtividade}

Retorno à produtividade é freqüentemente base para medida de recuperação de um trauma ou doença e é particularmente relevante quando se examina o ônus individual ou social do trauma, onde a maioria dos atingidos ế o adulto jovem.

Nas 83 vítimas das quais conhecia-se a evolução de 1 ano analisou-se o retorno à produtividade. Para tal análise inicialmente essas vítimas foram classificadas segundo sua ocupação principal, sendo apresentada sua distribuição segundo essa categorização na Tabela 2. 
Tabela 2 - Distribuição das vítimas com evolução de 1 ano segundo sua ocupação principal. São Paulo, 1993/94.

\begin{tabular}{|c|c|c|}
\hline Ocupação principal & $\mathbf{n}^{\circ}$ & $\%$ \\
\hline $\begin{array}{l}\text { remunerada } \\
\text { estudante e ocupação remunerada } \\
\text { estudante } \\
\text { desempregado } \\
\text { atividades do lar } \\
\text { aposentado }\end{array}$ & $\begin{array}{l}47 \\
13 \\
09 \\
09 \\
03 \\
02\end{array}$ & $\begin{array}{l}56,7 \\
15,7 \\
10,8 \\
10,8 \\
3,6 \\
2,4\end{array}$ \\
\hline TOTAL & 83 & 100 \\
\hline
\end{tabular}

A maioria das vitimas $(56,7 \%)$ tinha ocupação remunerada como atividade principal. Além disso, entre os 22 estudantes, a maioria (13) também tinha atividade remunerada. Devido ao critério de inclusão na população de vítimas de até 60 anos, a menor freqüência foi a de aposentados $(2,4 \%)$. $O$ desemprego ocorreu em $10,8 \%$ dos avaliados, sendo tal freqüência compatível com a taxa de desemprego da região no período. Segundo informações da Fundação Sistema de Análise de Dados (SEADE), a taxa de desemprego na Grande São Paulo, no primeiro semestre de 1993 , foi em média de $15,4^{12}$.

Na época do evento traumático, $72(86,8 \%)$ pessoas apresentavam atividades ocupacionais e foram analisadas quanto a seu retorno à produtividade. Para subsidiar tal análise, agrupou-se os traumatizados, segundo sua condição em relação a ocupação principal em 3 categorias conforme descrito na metodologia e a seguir discriminadas.

- Atividade interrompida: nesta categoria foram incluídos indivíduos que ap6s o trauma mantinham-se afastados do trabalho remunerado exercido regularmente e estudantes e pessoas envolvidas em afazeres do lar afastadas de suas atividades. Quando o indivíduo mantinha ocupação remunerada e era estudante, s6 era incluído nessa categoria se afastado das duas atividades.

- Alterada devido ao trauma: foram incluídos nesta categoria indivíduos com mudança de ocupação conseqüente às condições pós-traumáticas; retorno à ocupação anterior sem conseguir o mesmo nível de desempenho; retorno à ocupação anterior reduzindo o tempo dedicado ao seu desempenho; desempregado apos trauma, porém mantendo trabalho remunerado exercido de forma irregular; estudantes e pessoas envolvidas em atividades do lar que retornaram a essas atividades sem conseguir manter o mesmo desempenho. Fizeram parte também desse grupo indivíduos que mantinham tanto ocupação remunerada como atividades escolares, caso estivessem afastados de uma dessas duas atividades, ou ainda, se numa delas ou ambas apresentassem alteração de desempenho.

- Retorno com o mesmo desempenho: neste grupo foram incluídos indivíduos que retornaram a sua ocupação principal com o mesmo nível de desempenho. 
Apresenta-se na Tabela 3 as vítimas que mantinham atividades produtivas segundo ocupação principal e sua condição em relação a essa atividade após o trauma.

Tabela 3 - Distribuição das vítimas com atividade produtiva no período anterior ao trauma segundo ocupação principal e condição ocupacional aos 12 meses após TCE. São Paulo, 1993/94.

\begin{tabular}{|c|c|c|c|c|c|c|c|c|c|c|}
\hline \multirow[b]{2}{*}{$\begin{array}{l}\text { Condição } \\
\text { ocupacional }\end{array}$} & \multicolumn{2}{|c|}{ Remunerada } & \multicolumn{2}{|c|}{$\begin{array}{l}\text { Atividades } \\
\text { do lar }\end{array}$} & \multicolumn{2}{|c|}{$\begin{array}{c}\text { Remunerada } \\
+ \\
\text { estudante }\end{array}$} & \multicolumn{2}{|c|}{ Estudante } & \multicolumn{2}{|c|}{ TOTAL } \\
\hline & $n^{0}$ & $\%$ & $\mathrm{n}^{0}$ & $\%$ & $n^{\circ}$ & $\%$ & $n^{\circ}$ & $\%$ & $n^{\circ}$ & $\%$ \\
\hline -atividade interrompida & 11 & 15,3 & -- & - & 01 & 1,4 & -. & -- & 12 & 16,7 \\
\hline $\begin{array}{l}\text {-alterada devido ao } \\
\text { trauma }\end{array}$ & 05 & 6,9 & 01 & 1,4 & 07 & 9,7 & 01 & 1,4 & 14 & 19,4 \\
\hline $\begin{array}{l}\text {-retorno com o mesmo } \\
\text { desempenho }\end{array}$ & 31 & 43,1 & 02 & 2,8 & 05 & 6,9 & 08 & 11,1 & 46 & 63,9 \\
\hline TOTAL & 47 & 65,3 & 03 & 4.2 & 13 & 18,0 & 09 & 12,5 & 72 & 100,0 \\
\hline
\end{tabular}

Ao completar 1 ano, a maioria (63,9\%) havia retornado d produtividade com desempenho semelhante e somente uma minoria $(16,7 \%)$ encontrava-se afastada das atividades que normalmente exercia. Vítimas que tinham alteração na ocupação principal, eram nesse período $19,4 \%$.

No geral, o percentual de vítimas que retornou a produtividade aos 12 meses foi de $83,3 \%(19,4 \%+63,9 \%)$ quando vítimas que retornaram a ocupação principal mantendo o mesmo nível de desempenho ou com limitações, foram consideradas produtivas.

Resultado semelhante foi encontrado por MACKENZIE et $\mathrm{al}^{28}$ no seguimento de vítimas de trauma em geral. Eles verificaram que apos 1 ano da alta hospitalar, $17,0 \%$ dos traumatizados em seguimento ainda encontravam-se incapazes de trabalhar, ir à escola ou realizar as atividades do lar. Resultados menos favoráveis foram obtidos por MORRIS et $\mathrm{al}^{32}$, quando analisam vítimas de trauma cujo custo da internação superou a 100.000 dólares. Nesses indivíduos o retorno à produtividade, avaliado pelo menos 1 ano pós-trauma, era de $54,5 \%$. Entretanto, nesse mesmo estudo, ao se analisarem separadamente as vítimas de TCE observou-se que o retorno à ocupação principal foi de $72,0 \%$.

No período de 1 ano, todas as pessoas envolvidas em atividades do lar ou somente estudantes antes do trauma haviam retornado à produtividade. $O$ mesmo não ocorreu com os indivíduos que mantinham atividades remuneradas. Dos 13 estudantes que trabalhavam, 1 continuava afastado e das 47 vítimas com trabalho remunerado 11 continuavam com atividade interrompida, atingindo assim proporção de aproximadamente 1 afastado para cada 5 indivíduos. 
Ainda que o periodo de 1 ano possa ser considerado suficiente para pelo menos iniciar o preparo dos afastados do trabalho remunerado para outra função compativel com sua incapacidade ${ }^{27}$, nenhuma das 12 vítimas afastadas, encontrava-se em tal situação. Além disso, 2 vítimas, que não tinham possibilidade de retorno a sua função anterior, queixavam-se também de ausência de apoio social para preparo em outra área de atuação. Outro sentimento expresso pelos afastados era a vontade de retornar ao trabalho, contrapondo-se ao receio de desemprego em conseqüência da incapacidade de manter o mesmo nível de desempenho em sua função.

Considerando-se todos individuos que mantinham atividade remunerada, isto é, estudantes ou não que apresentavam esse tipo de ocupação, tinha-se 60 vítimas. Destas, no periodo de 1 ano, 12 não haviam retornado ao seu trabalho remunerado, perfazendo percentual de $20,0 \%$.

Os dados apresentados permitem delinear o padrão de recuperação das vítimas de TCE aos 12 meses pós-trauma. Nesse padrão, observa-se que a maioria das vítimas apresentam boa recuperação $(77,2 \%)$ e retornam à produtividade $(83,3 \%)$.

Ainda que os 6 primeiros meses possa ser considerado como intervalo de tempo de estabilização da curva de recuperação de saída funcional ${ }^{10,11,15,34,47} \mathrm{e}$ uma época em que a maioria das vítimas retorna às atividades que exercia anteriormente ao trauma, deficiências ou incapacidades residuais permanecem por períodos superiores a 1 ano as quais interferem na capacidade da vítima exercer papéis e funções que dela são esperados.

Embora o evento traumático geralmente leve somente segundos para ocorrer, seus efeitos permanecem por longos períodos na vítima e seus familiares, sendo poucos eventos na vida, que como o TCE, podem precipitar mudanças tão acentuadas em papéis, relações e objetivos de suas vítimas ${ }^{45}$.

A necessidade de assistência desses traumatizados permanece, portanto, por longos períodos após TCE, e vão além da necessidade de auxílio para retomar suas capacidades funcionais. Controle dos riscos em potencial e da sintomatologia apresentada, suporte para reinserção social à medida que dificuldades se estabeleçam, apoio para estabilização emocional de indivíduo e família e obtenção de informações fatuais são importantes demandas de assistência apresentada pelos traumatizados as quais deveriam ser atendidas com recursos e por equipe multiprofissional de saúde preparada para esse atendimento ${ }^{6,45}$. A ausência de tais condições de assistência, para o atendimento de muitas vítimas traumatizadas, conduz a maior dimensão do problema, tanto a indivíduos e famílias, como aumentam a magnitude das conseqüências do TCE na sociedade.

De fato, o TCE afeta a vítima pessoalmente, interpessoalmente e o seu sistema social. Além disso, seu impacto financeiro está duplamente presente. Por um lado, pelo custo hospitalar seguido de longo período de necessidade de suporte do sistema de saúde para reabilitação e por outro, compondo esse impacto estão as semanas e anos de perda de produ tividade. Claramente, as ocorrências do TCE e suas conseqüências têm atingido proporções epidêm icas justificando atenção como um grande problema de saúde da sociedade. 
SOUSA, R. M. C. de; KOIZUMI, M. S. Traumatic Brain Injury patients 1 year after trauma.

Rev.Esc.Enf.USP, v.30, n.3, p.484-500, dec. 1996.

A prospective longitudinal study of traumatic brain injury (TBI) patients was conduced to identify the recovery pattern 1 year after trauma. Patients with all levels of injury and age between 12 and 60 years were observed. They were analyzed using the eight-point Glasgow Outcome Scale (GOS) as well as their return to productivity in that period. The majority of victims $(77,2 \%)$ made good recovery, that is (GOS $=0$ or 1) and complete recovery or GOS 0 was achieved in $38,6 \%$ of then. However at the 1 year mark, $22,8 \%$ of the victims showed disabilities. Overall, victims had returned to productivity in that period $(83,3 \%)$ but $19,4 \%$ of them have had changes in their productivity and $16,7 \%$ didn't return to your job.).

UNITERMOS: Traumatic brain injury. Recovery pattern after trauma. Glasgow Outcome Scale

\section{REFERÊNCIAS BIBLIOGRÁFICAS}

01. THE ABBREVIATED Injury Scale : 1990 revision. Des Plaines, Association for the Advancement of Automotive Medicine, 1990.

02. ACORN, S. ; ROBERTS, E. Head injury: impact on the wives. J. Neurosci. Nurs., v.24, n.6, p.324-8, 1992.

03. BRASIL. Ministério da Saúde. Secretaria Nacional de Açðes Básicas de Saúde. Estatísticas de mortalidade: Brasil, 1989. Brasília, Centro de Documentaçăo, 1993

04. BRICOLO, A. et al. Prolonged posttraumatic unconsciousness: therapeutic assets and liabilities. J. Neurosurg., v.52, p.625.34, 1980.

05. BROOKS, D. N. et al. Cognitive sequelae of severe head injury in relation to the Glasgow Outcome Scale. J. Neurol. Neurosurg. Psychiatry, v.49, n.5, p.549.53, 1986.

06. CAMPBELL, C. H. Needs of relatives and helpfulness of support groups in severe head injury. Rehabil. Nurs., v. 13, n.6, p.320-5, 1988.

07. CAREY, R. G.; POSAVAC, E. J. Rehabilitation program evaluation using a Revised Level of Rehabilitation Scale (LORS-II). Arch. Phys. Med.Rehabil., v.63, n.8, p.367-70, 1982.

08. CHOI, S. C. et al. Prediction tree for severely head-injured patients. J. Neurosurg., v.75, n.2, p.251.5, 1991.

09. DALOSSI, T.; KOIZUMI, M. S. Estudo comparativo da gravidade do trauma de pacientes com ou sem traumatismo crânio-encefálico. Rev. Bras. Neurol. v.30, n.6, p.181-9, 1994.

10. DIKMEN, S. et al. Neuropsychological recovery in head injury. Arch. Neurol., v.40, p.333-8, 1983

11. FRUTIGER, A et al. Five years' follow-up of severely injured ICU patients. J.Trauma, v.31, n.9, p.1216-26, 1991.

12. FUNDAÇĀO SISTEMA ESTADUAL DE ANÁLISES DE DADOS - SEADE. Taxa de desemprego na grande Sao Paulo em 1993. São Paulo, s.d. IMimeografado/. 
13. GOUVIER, W. D. et al. Reliability and validity of the disability rating scale and the levels of Cognitive Functioning Scale in monitoring recovery from severe head injury. Arch. Phys. Med. Rehabil., v.68, p.94-7, 1987.

14. GROSWASSER, Z.; SAZBON, L. Outcome in 134 patients with prolonged posttraumatic unawareness. J. Neurosurg., v.72, p.81-4, 1990.

15. HALL, K. et al. Glasgow Outcome Scale and Disability Rating Scale: comparative usefulness in following recovery in traumatic head injury. Arch. Phys. Med. Rehabil. v.66, n.1, p.35-7, 1985.

16. HAMILTON, L. W.; CREASON, N. S. Mental status and functional abilities: change in institutionalized elderly women. Nurs. Diagn., v.3, n.2, p.81-6, 1992.

17. HEIDEN, J. S. et al. Severe head injury: clinical assessment and outcome. Phys. Ther., v.63, n. 12, p. $1946-51,1983$

18. JANE. J. A.; RIMEL, R. W. Prognosis in head injury. Clin Neurosurg., v.29, p.346.52, 1982.

19. JENNETT, B. Assessment of the severity of head injury. J. Neurol. Neurosurg., Psychiatry, v.39, n.7, p.647-55, 1976.

20. JENNETT, B. Predictors of recovery in evaluation of patients in coma. Adv. Neurol. v.22, p.129-35, 1979.

21. JENNETT, B.; BOND, M. Assessment of outcome after severe brain damage. Lancet, v.1, n. 7905, p. $480-4,1975$.

22. JENNETT, B. et al. Severe head injuries in three countries. J. Neurol. Neurosurg. psychiatry, v.40, n.3, p.291.8, 1977.

23. JENNETT, B. et al. Disability after severe head injury: observations on the use of the Glasgow Outcome Scale. J.Neurol Neurosurg. Psychiatry, v.44, n.4, p.285-93, 1981.

24. KIVIOJA, A. H. et al. Is the treatment of the most severe multiply injured patients worth the effort? - a followup examination 5 to 20 years after severe multiple injury. J. Trauma, v.30, n.4, p.480-3, 1990.

25. KRAUS, J. F. Epidemiology of head injury. In: COOPER,P.R. Head injury, 3.ed. New York, Williams \& Wilkins, 1993. cap.1, p.1-25.

26. LEVATI, A et al. Prognosis of severe head injuries. J.Neurosurg., v.57, n.6, p.779-83, 1982.

27. MACKENZIE, E. J. et al. Factors influencing return to work following hospitalization for traumatic injury. Am. J. Public. Health, v.77, n.3, p.329-34, 1987.

28. MACKENZIE, E. J. et al. Functional recovery and medical costs of trauma: an analysis by type and severity of injury. J.Trauma, v.28, n.3, p.281-97, 1988.

29. MANREZA,L.A. Atendimento ao paciente com traumatismo craniencefálico. In: NITRINI,R (ed.) . Condutas em neurologia, São Paulo, HC/FMUSP, 1989, cap.41. p. 156-63.

30. MANREZA, L. A.; RIBAS, G. C. Traumatismos craniencefálicos. In: NITRINI, R.; BACHESCHI, L. A. A Neurologia que todo médico deve saber. São Paulo, Maltese, 1991. cap.8, p.149.66.

31. MASET, A. et al. Epidemiologic features of head injury in Brazil. Arq. Bras. Neurocirurg, v. 12, p.293-302, 1993. 
32. MORRIS, J. A. et al. Trauma patients return to productivity. J Trauma. v.31, n.6. p.827-34, 1991.

33. ORGANIZAÇÃO MUNDIAL DE SAÚDE. Classificação Internacional das Deficiências, Incapacidades e Desvantagens (Handicaps): um manual de classificaçăo das consequências das doenças. Lisboa, 1989.

34. RAPPAPORT, M. et al. Disability rating scale for severe head trauma: coma to community. Arch. Phys. Med. Rehabil.. v.63, n.3, p.118-23, 1982.

35. RAPPAPORT, M. et al. Head injury outcome up to ten years later. Arch. Phys. Med. Rehabil., v.70, n.13, p.885-92, 1989.

36. RHODES, M. et al. Quality of life after the trauma center. J.Trauma, v.28, n.7, p.931-8, 1988.

37. RIMEL, $R$. W. et al. Moderate head injury: completing the clinical spectrum of brain trauma. Neurosurgery. v.11, n.3. p.344-51, 1982.

38. ROSS, A. M. et al. Prognosticators of outcome after major head injury in the elderly. J.Neurosci. Nurs., v.24, n.2, p.88-93, 1992.

39. SEID, M. E. O Brasil na contramão. Folha de São Paulo. São Paulo, 7 mar. 1994. p.3-2.

40. SOUSA, R. M. C. Padrão de recuperaçăo das vítimas de trauma crânio-encefálico aos 6 meses e 1 ano. Sá Paulo,1995, 104p. Tese (doutorado) - Escola de Enfermagem, Universidade de Sæo Paulo.

41. SPETTELL, C. M. et al. Time of rehabilitation admission and severity of trauma: effect on brain injury outcome. Arch. Phys. Med. Rehabil., v.72, n.5, p.320-5, 1991.

42. STROHMYER, L. L. et al. Adaptation six months after multiple trauma: a pilot study. J. Neurosci. Nurs., v.25, n.1, p.30-7, 1993.

43. TATE. R. L. et al. Impairment after severe blunt head injury: the results from a consecutive series of 100 patients. Acta Neurol. Scand v.79, n.2, p.97-107, 1989.

44. TEASDALE, G.; JENNETT, B. Assessment of coma and impaired consciousness. Lancet, v. $2, \mathrm{n} .7872, \mathrm{p} .81 .3,1974$.

45. TESTANI-DUFOUR, L. et al. Traumatic brain injury: a familiy experience. J. Neuroci. Nurs., v.24, n.6. p.317-23, 1992.

46. VOLLMER, D. G. Prognosis and outcome of severe head injury. In: COOPER, P. R. Head injury. 3.ed. New York, Williams \& Wilkins, 1993. cap.23, p.553-81.

47. VOLLMER, D. G. et al. Age and outcome following traumatic coma: why do older patients fare worse? J. Neurosurg., v.75, p.S37-49, 1991.

48. WARREN, J. B.; PECK, E. A. Factors which influence neuropsychological recovery from severe head injury. J. Neurosurg. Nurs.. v.16, n.5, p.248-52, 1984.

49. YOUNG, B. et al. Early prediction of outcome in head-injured. J. Neurosurg., v.54, p. 300-3, 1981. 